\title{
Selection Method of Surfactants for Chemical Enhanced Oil Recovery
}

\author{
Roland Nagy ${ }^{*}$, Rubina Sallai1, László Bartha1, Árpád Vágó ${ }^{2}$ \\ ${ }^{1}$ Department of MOL-Hydrocarbon and Coal Processing, University of Pannonia, Veszprém, Hungary \\ ${ }^{2} \mathrm{MOL}$ Plc. Research and Business Development, Budapest, Hungary \\ Email: ${ }^{\text {nroland@almos.uni-pannon.hu }}$
}

Received 16 February 2015; accepted 3 March 2015; published 6 March 2015

Copyright (C) 2015 by authors and Scientific Research Publishing Inc.

This work is licensed under the Creative Commons Attribution International License (CC BY). http://creativecommons.org/licenses/by/4.0/

(c) $\underset{\mathrm{EY}}{\mathrm{G}}$ Open Access

\begin{abstract}
Alternative energy sources have not yet presented suitable to meet the energy demand of the world; therefore crude oil will play furthermore an essential role in the energy consumption in the future. EOR is a challenging field for several scientific disciplines. The number of patents highlights the importance of this area. Most of the publications label that the target of the chemical processes is the reduction of IFT between the displacing liquid and oil phase. Based on the results in the last two decades the surfactants and flow modifier type polymers have shown more potential for a higher efficiency of the EOR than in any other methods. The aim of this work was to develop different surfactant testing methods that are capable to characterize the most important surfactant properties separately and to evaluate their combined or complementary effects. There was no correlation between the specific characteristics. But the weak correlation was detected by using the CE complex evaluation method. Further improvements could contribute significantly to improve the selectivity of the new experimental non-ionic surfactants for EOR applications.
\end{abstract}

\section{Keywords}

EOR, Method Development

\section{Introduction}

Alternative energy sources have not yet presented suitable to meet the energy demand of the world; therefore crude oil will play furthermore an essential role in the energy consumption in the future. Considering the fact that the easily recoverable oil is running out and much oil remains in the reservoir after conventional methods

\footnotetext{
${ }^{*}$ Corresponding author.
}

How to cite this paper: Nagy, R., Sallai, R., Bartha, L. and Vágó, Á. (2015) Selection Method of Surfactants for Chemical Enhanced Oil Recovery. Advances in Chemical Engineering and Science, 5, 121-128. 
have been exhausted, the implementation of Enhanced Oil Recovery has become crucial to guarantee a continuing crude oil supply [1].

The Enhanced Oil Recovery (EOR) technology covers the injection of specific type of a fluid or fluids into the reservoir by several methods (e.g.: chemical, thermal and microbial). The injected fluid promotes to dislocate of crude oil toward the producing well. Besides, the injected fluids interact with the reservoir rock/oil system and generate advantageous conditions for oil recovery. These interactions incorporate lowering the interfacial tension (IFT), improving the flow properties, modify wettability and help developing preferential phase behaviour. As a consequence of the interactions, physical and chemical mechanisms can occur, as well as the formation of thermal energy [2]-[5].

EOR is a challenging field for several scientific disciplines. The number of patents highlights the importance of this area. Most of the publications label that the target of the chemical processes is the reduction of IFT between the displacing liquid and oil phase. Based on the results in the last two decades the surfactants and flow modifier type polymers have shown more potential for a higher efficiency of the EOR than in any other methods [6] [7].

Chemical flooding of oil reservoirs could be the one of the most successful method to increase oil recovery rate of the depleted reservoirs. The research and developing projects of the EOR surfactants are very costly due to the expensive field tests. Numerous screening test methods have been elaborated to reduce the costs and to estimate the potential efficiency of the tested surfactant compositions [3] [7] [8].

However, the previous experiments showed that the surfactant composition has to be effective under different and often extreme conditions, which are complicated to be modelled in the laboratories. There is no generally accepted test method for the selection of surfactants. Therefore, the further development of screening methods remains important.

The aim of this work was to develop different surfactant testing methods that are capable to characterize the most important surfactant properties separately and to evaluate their combined or complementary effects.

\section{Materials}

\section{Preparation of the Surfactant and Surfactant-Polymer Solutions}

For the preparation of the experimental colloid solutions one anionic (MOLANIONIC) and two nonionic (PENONIONIC I and II) surfactants were used, that were developed and produced by cooperative research groups of University of Pannonia and Hungarian Oil and Gas Company (MOL Plc). Furthermore partially hydrolysed synthetic polyacrylamide was applied as a flow modifier type polymer.

The polymer-surfactant solutions were prepared by using brine, derived from the petroleum reservoir of Algyő (Hungary).

The surfactant packages were prepared of the following components (detailed in Table 1).

The flow modifier type polymer (PAM type sulfonated co-polymer) was used in a concentration of $1000 \mathrm{ppm}$. The total concentration of the surfactant packages in the mixtures was 15,000 ppm.

\section{Methods}

A complex laboratory screening method was developed to definite the properties of EOR type surfactants by the application of the following methods to determine the surface-active properties and also to study the possibility of the complex evaluation of the measurement data.

\subsection{The Investigation of the Emulsifying Efficiency (EE)}

In this method first the $50 \%$ of brine containing $1.5 \%$ of the emulsifier packages and $0.1 \%$ polymer and $50 \%$ of model oil (crude oil) should be shaken together at room temperature over 7 complete translation cycles. After 30 minutes long storage the volume of the formed phases should be measured. Thereafter 4 hours of storage at $80^{\circ} \mathrm{C}$ the volumes of the various phases should be determined again. The amount of aqueous phase and the emulsionand oil phases are given in volume\% related to the total liquid volume.

The maximum difference between the parallel results was in the interval of $\pm 2 \%$. 
Table 1. Composition of the surfactants and surfactant-polymer mixtures.

\begin{tabular}{ccc}
\hline Symbols of package compositions & ST-marked mixtures & SM-marked mixtures \\
\hline Components & \multicolumn{2}{c}{ Ratio of components, m/m\% } \\
\hline MOLANIONIC & 55 & 60 \\
PENONIONIC I & 25 & 40 \\
PENONIONIC II & 20 & - \\
\hline
\end{tabular}

\subsection{Determination of the Oil Displacement Effect (ODE) by Thin Layer Chromatography (TLC)}

The complex surface activity method has not been reported in the specific publications, which could be the extent to porous surfaces characterized by washing efficiency of the oil. It was developed a method for oil flushing effect, that based on the foundations of thin layer chromatography of organic chemical analysis has been used.

The first step of the test procedure is the preparation of the thin layer. First the glass slab should be washed with water and n-heptane and let dry. Thereafter the slab should be plunged into chloroform-real core sand dispersion and let dry for 20 minutes. Then $4 \mu \mathrm{l}$ of crude oil should be spotted on the thin layer at $2 \mathrm{~cm}$ from the base of the glass slab. Then the sample should be run by $\mathrm{pH}$ adjusted (between 8.0 - 8.5) brine containing $1 \%$ of the emulsifier mixture at $80^{\circ} \mathrm{C}$ for 3 hours. The drying of the thin layer should be carried out at room temperature after 24-hour storage. The oil displacement efficiency (ODE) of the surfactant composition can be evaluated by the distance from the centre of the oil spot to the upper edge of the spot measured in mm. The layers after the 3-hours run-time should be photographed with a digital camera, and the data should be recorded and stored in a database.

The maximum difference between the parallel results was in the interval of $\pm 10 \%$.

\subsection{Determination of Interfacial Tension (IFT)}

The spinning drop method (rotating drop method) is one of the methods used to measure the oil-water interfacial tension. The measurements should be carried out in a rotating horizontal tube, which contains a dense fluid, and drop of a less dense liquid should be placed inside the fluid. Till the rotation of the horizontal tube creates a centrifugal force towards the tube walls, the liquid drop will start to deform into an elongated shape; this elongation stops when the interfacial tension and centrifugal forces are balanced. The surface tension between the two liquids (for bubbles: between the fluid and the gas) can then be derived from the shape of the drop at this equilibrium point. A device used for such measurements is called a "spinning drop tensiometer".

The spinning drop method is usually preferred for the accurate measurements of surface tensions below $10^{-2}$ $\mathrm{mN} / \mathrm{m}$. It refers to either using the fluids with low interfacial tension or working at very high angular velocities.

The maximum difference between the parallel results was in the interval of $\pm 5 \%$.

\subsection{Oil Displacement Test (ODT)}

The special core flooding tests were performed by a test method simulated the processes in the high-temperature oil reservoirs. The crude oil was originated from Algyö, Hungary. Testing temperature was $80^{\circ} \mathrm{C}$. The tests were performed in a core with inner diameter of $2.5 \mathrm{~cm}$ and length of $30 \mathrm{~cm}$. The heterogeneousness (homogeneity) of the formation was simulated by consolidated core sample.

Surfactant-polymer flooding has been successfully used to recover waterflood residual oil with acidic crude oils in laboratory-scale experiments. The additional oil recovery was determined, that was related to residual oil saturation after the water flooding [8].

The maximum difference between the parallel results was in the interval of $\pm 5 \%$.

\section{Results and Discussion}

The first aim in this work was to investigate how and by which selectivity can be used the laboratory test methods 
for characterization and evaluation of the experimental surfactant mixtures for EOR purposes.

In our study, as a model two different packages of experimental surfactants were examined (of two-and three-components). The suitability of these surfactant packages for EOR purposes has been studied by the presented test methods.

The three screening methods and the oil displacement test were used also for the complete classification of surfactant packages. The second aim of this work was to use the screening methods for the optimization the compositions of two surfactant packages consisting non-ionic type compounds with different molecular structure by variation of the ratio of the polar and nonpolar groups in the mixture of molecules obtained by reaction of fatty acids and a polyalkanol-amine.

\subsection{Investigation of SM-Surfactants Mixtures (Sequence of Two-Component)}

The polymer-surfactants mixtures should be used in a reservoir, where the temperature is between $80^{\circ} \mathrm{C}-100^{\circ} \mathrm{C}$. Therefore it was important to study the relationship between the high temperature surface-active properties and the composition of the non-ionic components used in the surfactant mixtures. The data of the SM-surfactants series are given in Table 2 and demonstrated in Figures 1-3.

Generally the additional oil yield in the Enhanced Oil Recovery is related to initial stock. In these experiments and after the water flooding the residual oil contents were between $40 \%-60 \%$.

The IFT region was published by the literature as an essential condition for EOR application, but the figure shows that there is no correlation between the displacement results and IFT values. However, previous researches proved, the IFT values below $0.075 \mathrm{mN} / \mathrm{m}$ were advantageous for Enhanced Oil Recovery application,

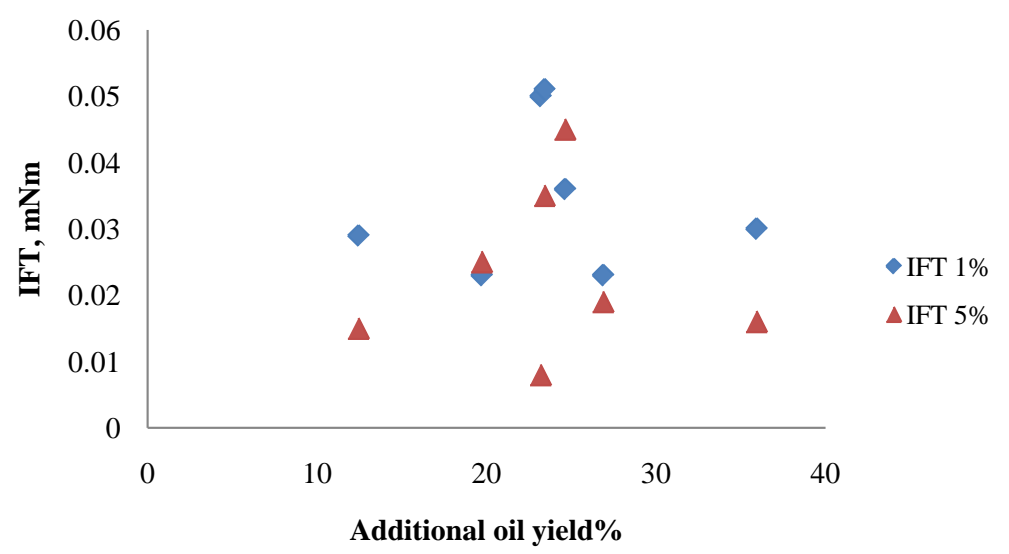

Figure 1. Relationship between the additional oil yield and the IFT.

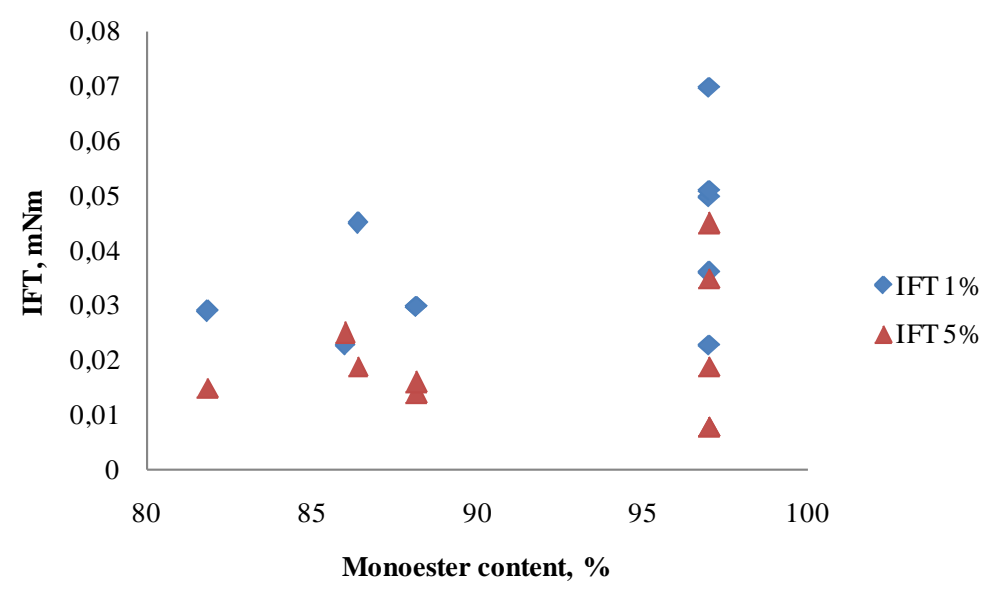

Figure 2. Relationship between the monoester content and IFT. 


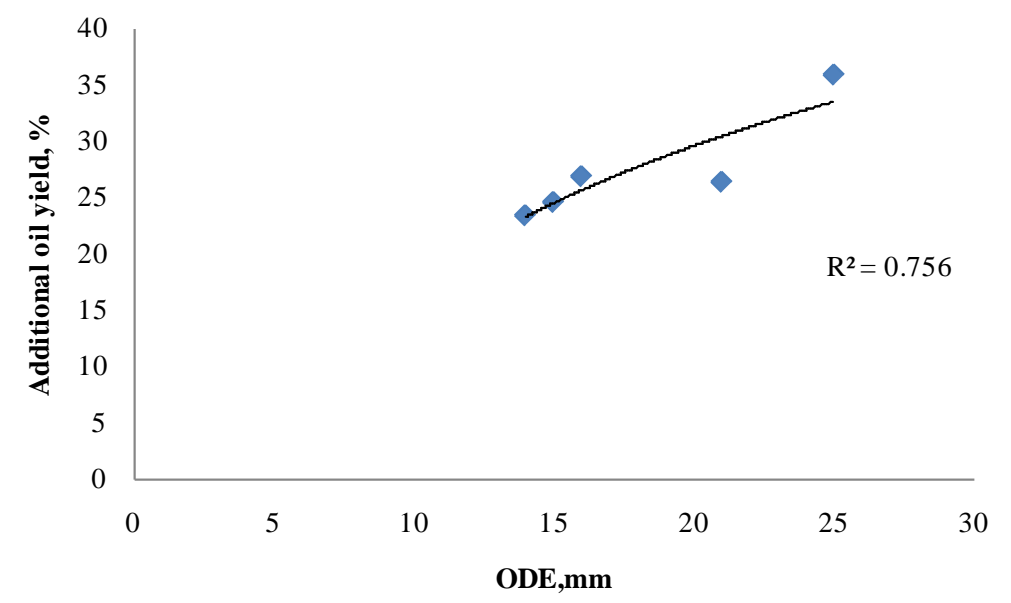

Figure 3. Relationship between the ODE and additional oil yield.

Table 2. Data of the surfactants and surfactant-polymer mixtures.

\begin{tabular}{|c|c|c|c|c|c|c|c|}
\hline & \multicolumn{2}{|c|}{$\begin{array}{l}\text { Composition of the nonionic surfactant } \\
\text { (PENONIONIC-1) }\end{array}$} & \multicolumn{2}{|c|}{$\begin{array}{l}\text { IFT, } \\
\text { mNm }\end{array}$} & \multirow{2}{*}{$\begin{array}{c}\mathrm{EE} \\
1 \mathrm{~h}, 80^{\circ} \mathrm{C}, \%\end{array}$} & \multirow{2}{*}{$\begin{array}{c}\text { ODE } \\
\mathrm{mm}\end{array}$} & \multirow{2}{*}{$\begin{array}{c}\text { Additional } \\
\text { oil yield } \\
\%\end{array}$} \\
\hline & Monoester, \% & Diester, \% & $1 \%$ & $5 \%$ & & & \\
\hline SM-1 & 88.17 & 4.1 & 0.03 & 0.016 & 100 & 25 & 35.94 \\
\hline SM-2 & 97.00 & 2.46 & 0.05 & 0.008 & 97.5 & 20 & 23.20 \\
\hline SM-3 & 81.85 & 16.9 & 0.029 & 0.015 & 10 & 15 & 12.45 \\
\hline SM-4 & 86.00 & 13.37 & 0.023 & 0.025 & 10 & 21 & 19.72 \\
\hline SM-5 & 78.93 & 15.12 & 0.039 & 0.028 & 50 & 16 & - \\
\hline SM-6 & 86.39 & 12.21 & 0.045 & 0.019 & 12.5 & 19 & - \\
\hline SM-7 & 97.00 & 2.46 & 0.051 & 0.035 & 95 & 14 & 23.42 \\
\hline SM-8 & 97.00 & 2.46 & 0.036 & 0.045 & 85 & 15 & 24.64 \\
\hline SM-9 & 97.00 & 2.46 & 0.023 & 0.019 & 40 & 16 & 26.89 \\
\hline
\end{tabular}

what criteria related to each sample had satisfied [8].

Between the monoester content and interfacial tension there was not clear tendency as well. The other presented methods (EE, ODE) are mainly effect on the mobility properties of the oil/water emulsion, which have been found appropriate in the measured range. Experimental results of each test methods were not correlated with the replacement characteristics.

It was found, if the additional oil yield values are over $20 \%$, than it can be evaluated as a successful recovery composition. Between the ODE and additional oil field a saturation curve type tendency was observed.

The additional oil yield method is considered to be the most important method of classification of surfactants for EOR. Therefore based on these results the ODE method could be also suitable and significantly cheap and fast method in the pre-selection process of the potential EOR surfactants

The higher diester content seemed advantageous to achieve an extreme low IFT value. Therefore the correlation could be used also for optimization of the composition of the non-ionic surfactant packages.

\subsection{Investigation of ST-Surfactants Mixtures (Sequence of Three-Component)}

The data of the ST-surfactants are given in Table 3. and demonstrated in Figure 4 and Figure 5. According to 


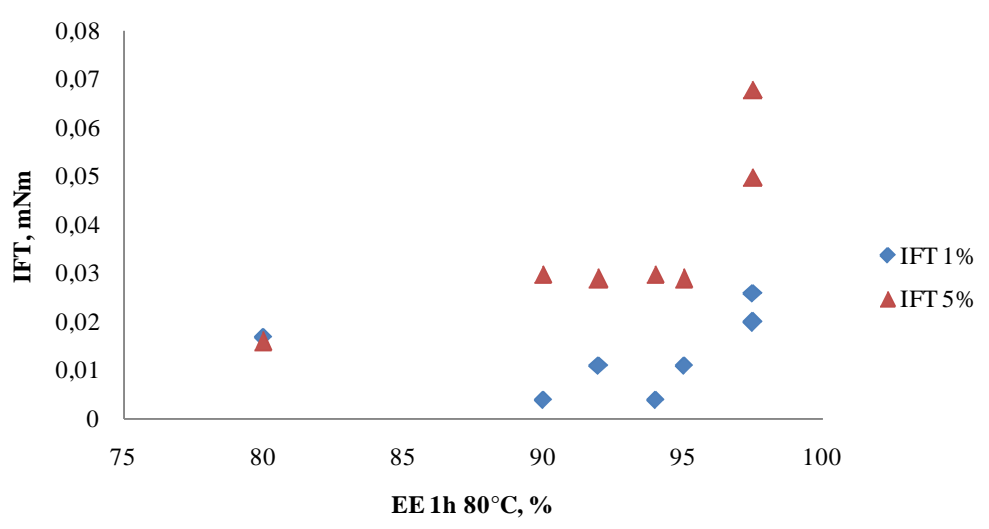

Figure 4. Relationship between the emulsifying effect and IFT.

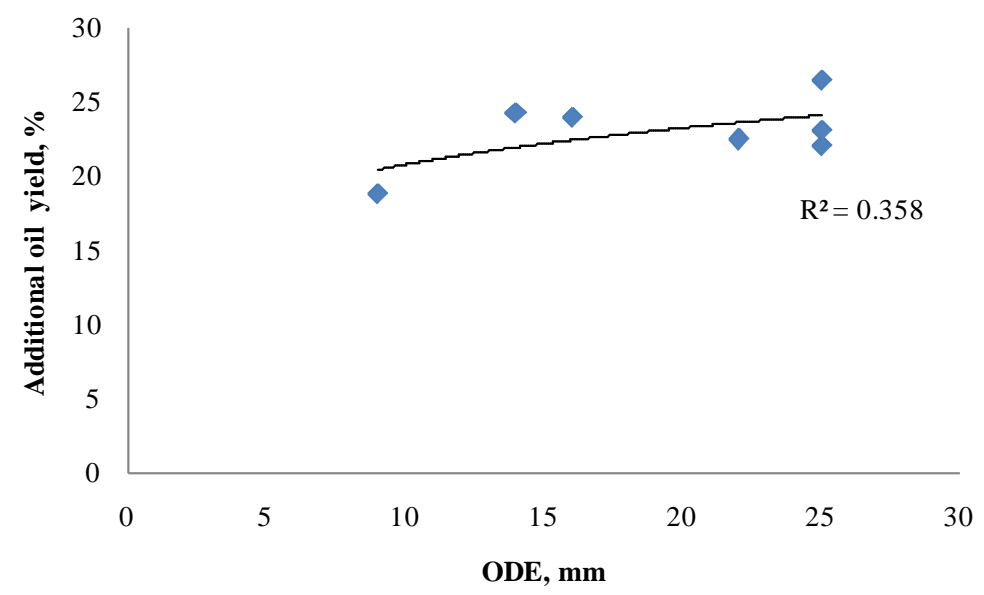

Figure 5. Relationship between the ODE and additional oil yield.

Table 3. Data of the surfactants and surfactant-polymer mixtures.

\begin{tabular}{rccccccc}
\hline & $\begin{array}{c}\text { Composition of the nonionic surfactant } \\
\text { (PENONIONIC-1) }\end{array}$ & \multicolumn{2}{c}{ IFT, mNm } & EE & ODE & $\begin{array}{c}\text { Additional } \\
\text { oil yield }\end{array}$ \\
\cline { 2 - 7 } & Monoester, \% & Diester, $\%$ & IFT 1\% & IFT 5\% & $1 \mathrm{~h} 80^{\circ} \mathrm{C}, \%$ & $\mathrm{~mm}$ & $\%$ \\
\hline ST-1 & 97.00 & 2.46 & 0.004 & 0.03 & 90 & 16 & 24.03 \\
ST-2 & 97.00 & 2.46 & 0.026 & 0.068 & 97.5 & 9 & 18.08 \\
ST-3 & 86.39 & 12.21 & 0.011 & 0.029 & 95 & 25 & 22.13 \\
ST-4 & 86.39 & 12.21 & 0.02 & 0.1 & 97.5 & 26 & 23.09 \\
ST-5 & 81.85 & 16.9 & 0.011 & 0.029 & 92 & 22 & 22.46 \\
ST-6 & 86.00 & 13.57 & 0.017 & 0.016 & 80 & 14 & 24.35 \\
ST-7 & 78.93 & 15.12 & 0.004 & 0.013 & 94 & 25 & 26.50 \\
\hline
\end{tabular}

the preliminary tests the ST surfactant mixtures seemed also appropriate for EOR purposes that were supported by numerous of measurement data.

The IFT values were found below $0.1 \mathrm{~m} \cdot \mathrm{Nm}$, therefore this series of the surfactant mixtures can be suitable as well for EOR application. Between the emulsifying effect and the interfacial tension there was not clear tendency 
too. It was also found, that the IFT method in itself is not enough for classification of surfactants for EOR.

On the other hand it was found, that the additional oil yield values were also over $20 \%$ that can be considered a successful oil recovery ratio. In addition between the ODE and additional oil yield values an increasing tendency was observed with a low level of significance.

\subsection{CE Calculation}

Based on the results of the single laboratory test methods it was supposed that the high replacement efficiency of the surfactant packages only by a well balanced combination of the surfactant properties can be achieved. That is why a complex evaluation system was introduced and studied.

For the combined evaluation (CE) of the three properties an evaluation-equation was created and used to characterize the potential efficiency of the experimental surfactant for EOR. According to the mathematical calculations by using the measurement the best approximated equation was defined by the next formula:

$$
\mathrm{CE}=\frac{\mathrm{ODE} \cdot 0.25 \cdot \mathrm{EE}}{\mathrm{IEF}^{2} \cdot 100}
$$

The calculated CE value of the series of the SM and ST surfactants in a function of additional oil yield related to residual oil shows Figure 6.

It was concluded, that by increasing the CE value a better additional oil yield could be estimated. In spite of the weak correlation it is supposed that by using also other complementary characteristics of the surfactant packages the estimation of oil recovery may be further improved. Figure 6 showed that in accordance with the general practice by using these experimental surfactant mixtures only a limited value (80\%) from the residual crude oil can be recovered by this SP method. Based on these results next enhancements are planned, which could further contribute to reduce the selection of new surfactants. By the use of the developed methods the number of performed measurement can be approximately one-tenth reduced.

\section{Conclusions}

The experimental results were summarized as follows:

- A new complex laboratory method was developed to estimate the efficiency of the experimental surfactant packages for EOR.

- Different levels of correlations have been obtained between the surface-active properties and the additional oil yield.

- Slightly correlation was found between the CE factor and the additional oil yield that could open new opportunities for cost reduction of the development.

- By these measurements the number of experimental surfactants and their mixtures can be reduced.

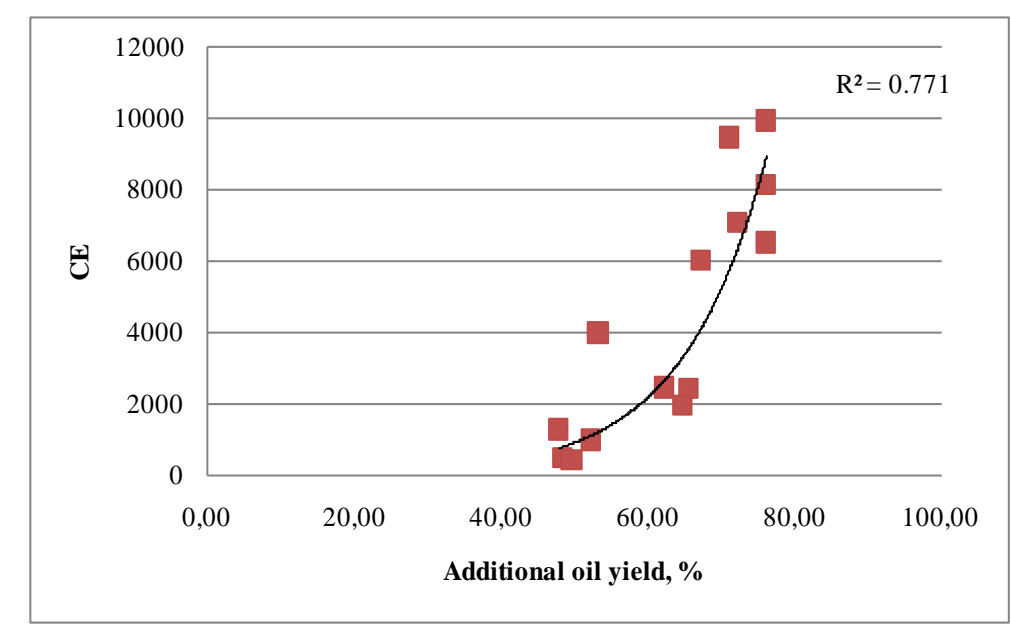

Figure 6. Relationship between the CE and additional oil yield (related to residual oil) in case of SM and ST-surfactants. 
There was no correlation between the specific characteristics. But the weak correlation was detected by using the CE complex evaluation method. Further improvements could contribute significantly to improve the selectivity of the new experimental non-ionic surfactants for EOR applications.

\section{References}

[1] Thomas, S. (2008) Enhanced Oil Recovery-An Overview. Oil \& Gas Science and Technology, 63, 9-19. http://dx.doi.org/10.2516/ogst:2007060

[2] Gaillard, N., Giovannetti, B. and Favero, C. (2010) Improved Oil Recovery Using Thermally and Chemically Protected Compositions Based on Co- and Terpolymers Containing Acrylamide. SPE Improved Oil Recovery Symposium, Tulsa, 24-28 April 2010, Document ID: SPE-129756-MS. http://dx.doi.org/10.2118/129756-MS

[3] Levitt, D.B. and Pope, G.A. (2008) Selection and Screening of Polymers for Enhanced-Oil Recovery. SPE Symposium on Improved Oil Recovery, Tulsa, 20-23 April 2008, Document ID: SPE-113845-MS.

[4] Zhong, C, Luo, P., Ye, Z. and Chen, H. (2009) Characterization and Solution Properties of a Novel Water-Soluble Terpolymer for Enhanced Oil Recovery. Polymer Bulletin, 62, 79-89. http://dx.doi.org/10.1007/s00289-008-1007-6

[5] Schramm, L.L. (2000) Surfactants: Fundamentals and Applications in the Petroleum Industry. Cambridge University Press, Cambridge.

[6] Myers, D. (2006) Surfactant Science and Technology. Wiley-Interscience, Hoboken.

[7] Jeirani, Z., Mohamed Jan, B., Si Ali, B., Noor, I.M., See, C.H. and Saphanuchart, W. (2013) Formulation, Optimization and Application of Triglyceride Microemulsion in Enhanced Oil Recovery. Industrial Crops and Products, 43, 6-14. http://dx.doi.org/10.1016/j.indcrop.2012.07.002

[8] Nasr-EI-Din, H.A., Hawkins, B.F. and Green, K.A. (1992) Recovery of Residual Oil Using the Alkali/Surfactant/ Polymer Process: Effect of Alkali Concentration. Journal of Petroleum Science and Engineering, 6, 381-401. http://dx.doi.org/10.1016/0920-4105(92)90064-8 